KfK 3586

September 1983

\title{
The Density Dependence of the Effective Alpha-Nucleon Force and Isoscalar Transition Rates of Nuclei
}

D. K. Srivastava, H. Rebel Institut für Kernphysik

Kernforschungszentrum Karlsruhe 
KERNFORSCHUNGSZENTRUM KARLSRUHE

Institut für Kernphysik

KfK 3586

THE DENSITY DEPENDENCE OF THE EFFECTIVE

ALPHA-NUCLEON FORCE

AND ISOSCALAR TRANSITION RATES OF NUCLEI

D.K. SRIVASTAVA ${ }^{+}$AND H. REBEL

Kernforschungszentrum Karslruhe $\mathrm{GmbH}$, Karlsruhe

+ Bhaba Atomic Research Institute, Calcutta, India 
Als Manuskript vervielfältigt

Für diesen Bericht behalten wir uns alle Rechte vor

Kernforschungszentrum Karlsruhe $\mathrm{GmbH}$ ISSN 0303-4003 
Abstract

Effects of the density-dependence of the $\alpha$-particle bound nucleon effective interaction on single-folding models of inelastic $\alpha$-particle scattering are studied, in particular in view of corrections of the isoscalar transition rates extracted by implicit folding procedures. The 1 -dependent correction. factors $C_{1}$ are calculated and tabulated for applications.

DIE DICHTEABHÄNGIGKEIT DER ALPHA-NUKLEON KRAFT UND ISOSKALARE ÜBERGANGSRATEN

Die Auswirkung der Dichteabhängigkeit der effektiven Wechselwirkung zwischen $\alpha$-Teilchen und Kernnukleonen auf EinfachFaltungsmodelle der unelastischen $\alpha$-Teilchenstreuung werden untersucht, insbesondere im Hinblick auf Korrekturen zu den isoskalaren Übergangsraten, die durch implizierte Faltungsmode11-Verfahren gewonnen werden. Die 1-abhängigen Korrekturfaktoren $C_{1}$ werden berechnet und im Anhang tabelliert. 


\section{Introduction}

The procedures currently used [1] for extracting isoscalar multipole transition rates from inelastic $\alpha$-particle scattering e.g. are implicitly or explicitly based on the assumption that the (real) form factor $U_{\text {if }}\left(r_{\alpha}\right)$ (coupling potential) can be understood as the result of a folding of the transition density $\rho_{\text {if }}=\left\langle i\left|\delta\left(\vec{r}-\vec{r}^{\prime}\right)\right| f\right\rangle$ with an effective $\alpha$-particle-bound nucleon interaction $v_{\text {eff }}^{\alpha N}\left(\vec{r}, \vec{r}_{\alpha}\right)$. Explicit folding model approaches require a rather detailed specification of $V_{e f f}^{\alpha N}$ which can be hardly done free from any arbitrariness, and the results derived may be affected by the particular choice of the effective interaction. This difficulty is avoided by implicit folding procedures, recently worked out in detail [2-4]. In a first step of the analysis of the measured cross sections the coupling potentials have been determined on a conventional optical-model basis, in some cases rather accurately by using flexible parametrizations of $U_{\text {if }}$ (thus minimizing constraints in the radial shapes) and including multiple excitation by coupled-channel calculations [2,4]. In a further step the transition moments and transition radii calculated for the resulting potentials are translated into corresponding quantities of the nuclear density distributions by use of simple mathematical properties $[5,6]$ (known as Satchler's theorem [6]) for the radial moments of folding integrals. The basic relation between the multipole moments e.g. of the potential $q_{1 m}^{u}$ and of the nucleon distribution $q_{1 m}^{\rho}$ is given by

$$
\frac{q_{1 m}^{u}}{J_{u}}=\frac{q_{1 m}^{\rho}}{J_{\rho}}
$$

with $J_{u}$ and $J_{\rho}$ being the volume integrals of the distributions

$$
U\left(\vec{r}_{\alpha}\right)=\int \rho(\vec{r}) V_{e f f}^{\alpha N}\left(\vec{r}_{\alpha}, \vec{r}\right) d \vec{r}
$$

and $\rho(\vec{r})$, respectively. It is mathematically correct as long as the function $V_{\text {eff }}^{\alpha \mathbb{N}}$ is central and independent from $\rho$. 
A density independent effective interaction, however, is only a first approximation. Refined analyses of $\alpha$-particle scattering have revealed the importance of density dependence, in particular when probing deeper into the nucleus with energies above $100 \mathrm{MeV}$. Quite recently Srivastava [10] has extended the concept of an implicit folding model analysis to a double folding basis and considered the consequences of the density dependence of the nucleon-nucleon interaction. The values of the deduced transition rates appear to be considerably affected, in particular for higher multipolarity transitions. In the present paper we consider the problem from the point of view of a single folding approach with a density dependent effective interaction $v_{\text {eff }}^{\alpha \mathbb{N}}\left(\vec{r}_{\alpha}, \vec{r}, \rho\right)$ and study the limits and corrections of the procedure proposed in ref. 2-4.

\section{Basic considerations}

We introduce the density dependence of the effective $\alpha$-boundnucleon interaction by a saturation factor $g(\rho)$, writing

$$
v_{\text {eff }}^{\alpha N}\left(\vec{r}_{\alpha}, \vec{r}_{,} \rho\right)=v_{\alpha N}^{\circ}\left(\left|\vec{r}-\vec{r}_{\alpha}\right|\right) \cdot g(\rho)
$$

Several alternative analytical forms for $g(p)$ are proposed (see ref. 11), and even the question which local density (the density to be used in 2.1) is not answered in a unique - way (see ref. 9). Following previous studies [11] we use here

$$
g(\rho)=\left[1-\gamma \rho^{2 / 3}(\vec{r})\right]
$$

with the assumption that the saturation of the effective interaction is due to the nucleon density at the site of the boundnucleon interacting with the $\alpha$-particle (positioned at $\vec{r}_{\alpha}$ ). In the context of explicit folding model studies of elastic $\alpha$-particle scattering it has been argued. [9] that this kind of local density (suggested by the relatively short range of $v_{\text {eff }}$ ) is not so well justified and that there are also contributions from the nucleon density $\rho\left(r_{\alpha}\right)$ at the site of the projectile. We think, 
however, that such refinements have only small influence on the integral quantities (radial moments) we are extracting by the implicit procedures. [4-6].

Introducing $v_{\text {eff }}^{\alpha N}(2.1)$ into the folding formula (1.2) and separating the density independent part of $\mathrm{V}_{\text {eff }}^{\alpha \mathrm{N}}$ leads to some kind of "effective density".

$$
\rho^{\prime}(\vec{r})=\rho(\vec{r})\left[1-\gamma \rho^{2 / 3}(\vec{r})\right]
$$

for which effective moments $q_{I m}^{\rho}$, volume integrals $J_{\rho}$, etc. can be defined, replacing $q_{I m}^{\rho}$ and $J_{\rho}$ in the above theorem (eq. 1.1). The volume integrals and ms radii of $\rho(r)$ and $\rho^{\prime}(x)$ are related [12] by

$$
J_{\rho}^{\prime}=A-Y K
$$

and

$$
\left\langle r^{2}\right\rangle_{\rho^{\prime}}=\left\langle x^{2}\right\rangle_{\rho}+[(\gamma K / A) /(1-\gamma K / A)]\left[\left\langle r^{2}\right\rangle_{\rho}-\left\langle r^{2}\right\rangle_{x}\right]
$$

where

$$
K=\int \rho^{5 / 3}(\vec{r}) d \vec{r}
$$

and

$$
\left\langle r^{2}\right\rangle_{x}=\int r^{2} \rho^{5 / 3}(\vec{r}) d \vec{r} / K
$$

For density-independent interactions $(\gamma \equiv 0) J_{\rho}$, and $\left\langle r^{2}\right\rangle_{\rho}$, reduce, of course, to $J_{\rho}$ and $\left\langle r^{2}\right\rangle_{\rho}$. But also in the case of a homogeneous density distribution, the density dependence of the effective interaction can be absorbed by renormalization factor of the density-independent strength, so that the relations

$$
\mathrm{J}_{\mathrm{u}}=\mathrm{A} \cdot \mathrm{J}_{\mathrm{V}}
$$

and

$$
\left\langle r^{2}\right\rangle_{\mathrm{u}}=\left\langle r^{2}\right\rangle_{\rho}+\left\langle r^{2}\right\rangle_{\mathrm{v}}
$$

known from the density independent folding model, are again valid. Since the density in the nuclear interior is very 
similar for all nuclei, an implicit folding model analysis of elastic $\alpha$-particle scattering, using the relation

$$
\Delta\left\langle r^{2}\right\rangle_{\mathrm{U}}^{\mathrm{AA}}=\Delta\left\langle r^{2}\right\rangle_{\rho}^{\mathrm{AA}}
$$

is well able to provide nuclear matter radii differences for neighboring nuclei [13]. Introducing the "defect moments"

$$
M_{1 m}=\int r^{1} Y_{I m} \rho^{5 / 3}(\vec{r}) d \vec{r}
$$

we extend the considerations to the multipole moments by

$$
\frac{q_{I m}^{\rho^{\prime}}}{J_{\rho^{\prime}}}=\frac{q_{I m}^{\rho}-\gamma M_{I m}}{A-\gamma K}
$$

Similarily the transition radius of the multipole transition 1 is

$$
R_{\operatorname{tr}}^{2}(1)=R_{t r}^{2}\left(1, \rho^{\prime}\right)+\frac{1}{3}(21+3)\left\langle r^{2}\right\rangle_{v}
$$

In order to get some feeling about the corrections, tab. 1 presents some numerical values for the relevant quantities, calculated for a (permanently) deformed Fermi distribution

$$
\rho(x)=\rho_{0} /[1+\exp ((x-c) / a)]
$$

with

$$
C(x)=C_{0}\left(1+B_{2} Y_{20}+B_{4} Y_{40}\right)
$$

and adopting the parameter values for the ${ }^{56} \mathrm{Fe}$ nucleus [14]

$$
\begin{array}{llll}
A & =56 & B_{2}=0.24 \\
C_{0}= & 3.98 \mathrm{fm} & B_{4}=0.03 \\
a & =0.53 \mathrm{fm} &
\end{array}
$$

\begin{tabular}{|ccccccc|}
\hline$\left\langle r^{2}\right\rangle_{\rho}$ & $q_{20}^{\rho}$ & $q_{40}^{\rho}$ & $K$ & $\left\langle r^{2}\right\rangle_{x}$ & $M_{20}$ & $M_{40}$ \\
{$[f m]$} & {$\left[\mathrm{fm}^{2}\right]$} & {$\left[\mathrm{fm}^{4}\right]$} & {$\left[\mathrm{fm}^{-2}\right]$} & {$\left[\mathrm{fm}^{2}\right]$} & {$\left[\mathrm{fm}^{0}\right]$} & {$\left[\mathrm{fm}^{2}\right]$} \\
\hline 3.70 & 63.7 & 241.7 & 12.7 & 10.5 & 12.9 & 42.6 \\
\hline
\end{tabular}

Tab. 1 Integral quantities of interest for a typical Fermi distribution

(in this case, the comments correspond to intrinsic multipole moments). 
3. Reduced transition moments of a Fermi distribution

Numerical calculations of the corrections of the theorem (1.1) due to the density dependence require a specification of the radial shape of $\rho(\vec{r})$ and a deformation prescription since we are discussing integral properties, it is reasonable to expect that the results are practically independent of the form of the density distribution as long as this form is leptodermous. In ref. 12 the relevant quantities $\mathrm{K},\left\langle r^{2}\right\rangle_{\mathrm{x}}$ for a (spherical) Fermi distribution $(k=1)$

$$
\rho(r)=\rho_{0}[1+\exp ((x-c) / a)]^{-k}
$$

are given in terms of $\varepsilon=a / c$. In first order in $\varepsilon$ the relation (2.6) is improved by

$$
\left\langle\mathrm{r}^{2}\right\rangle_{\mathrm{u}}=\left\langle\mathrm{r}^{2}\right\rangle_{\mathrm{\rho}}+\left\langle\mathrm{r}^{2}\right\rangle_{\mathrm{v}}+\frac{6}{5} \cdot \mathrm{c} \cdot \mathrm{a} \cdot \alpha \cdot \mathrm{A}_{1}
$$

with

$$
\begin{aligned}
& \alpha=\gamma \rho_{\circ}^{2 / 3} /\left(1-\gamma \rho_{o}^{2 / 3}\right) \\
& A_{1}(5 / 3)=0.759 \text { (see ref. 12) }
\end{aligned}
$$

showing that the correction is linear in $\mathrm{c}$ and $\mathrm{a}$.

Here we extend the calculations to the multipole transition moments of a deformed Fermi distribution with an angular dependent half-way radius

$$
c=c_{0}\left(1+\Sigma \alpha_{1 m} Y_{1 m}(\hat{r})\right)
$$

The reduced transition density, written in the form of a higher order ( $t$ ) vibrational model, is

$$
\rho_{1}(r)=\Sigma_{t} \rho_{1}^{(t)}(r)\left\langle I|| Q_{1}^{(t)}|| I^{\prime}\right\rangle
$$

with $\left\langle I|| Q_{1}^{(t)}|| I^{\prime}\right\rangle$ the reduced matrix elements ("deformation parameters") of the transition operators (built by the $\alpha_{1 \mathrm{~m}}$ ), and

$$
\rho_{1}^{(t)}=\frac{1}{t !} c_{0}^{t} \frac{\partial^{t}}{\partial c_{0}^{t}}
$$

We are interested in the reduced transition moments which are rather directly related to the reduced transition probabilities. We have to calculate

$$
q_{1}^{\rho^{\prime}}=\int \rho i(r) \cdot r^{1+2} d r=q_{1}-\gamma M_{1}
$$


Introducing the moments of the derivative expressions (3.5) up to $t=2$ for distributions of the type (3.1) with $k=1$ and $k=5 / 3$,

$$
\begin{aligned}
& y_{1}(\kappa)=c_{0} \int \frac{\partial \rho(\kappa)}{\partial c_{0}} r^{1+2} d r \\
& z_{1}(\kappa)=\frac{c_{0}^{2}}{2} \int \frac{\partial^{2}{ }^{2}(\kappa)}{\partial c_{0}^{2}} r^{1+2} d r
\end{aligned}
$$

we write

$$
\begin{aligned}
& q_{1}^{\rho^{\prime}}=\left\langle I|| Q_{1}{ }^{(1)}|| I^{\prime}\right\rangle \cdot Y_{I}(1)+\left\langle I|| Q_{I}{ }^{(2)}|| I^{\prime}\right\rangle \cdot z_{1}(1) \\
& \left.-\gamma \rho_{0}{ }^{2 / 3}\left(\left\langle I|| Q_{1}{ }^{(1)}|| I^{\prime}\right\rangle \cdot y_{I}(5 / 3)+\left\langle I|| Q_{1}{ }^{(2)}|| I^{\prime}\right\rangle \cdot z_{1}(5 / 3)\right)\right)
\end{aligned}
$$

Following ref. $15 Y_{I}(k)$ and $z_{1}(k)$ can be expanded in power series in $\varepsilon=a / c$. We adopt following definitions:

$$
I_{I}=\frac{\left\langle I|| Q_{1}{ }^{(2)}|| I^{\prime}\right\rangle / 2}{\left\langle I|| Q_{1}{ }^{(1)}|| I^{\prime}\right\rangle+(I+2) / 2 \cdot\left\langle I|| Q_{1}{ }^{(2)}|| I^{\prime}\right\rangle}
$$

$$
\begin{aligned}
\mathrm{H}_{1}(k)=1 & +\sum_{n=0}^{1}(-1)^{n+1} \frac{(1+2) !}{(1-n+1) !} A_{n+1}(k) \varepsilon^{n+1}\left(1-(n+1) I_{I}\right) \\
& +(-1)^{1}(1+2) ! A_{1+2}(k) \varepsilon^{1+2}
\end{aligned}
$$

with [15]

$$
A_{1}(1)=0, A_{2}(1)=\pi^{2} / 6, A_{3}(1)=0 \quad \text { etc. }
$$

$$
A_{1}(5 / 3)=0.759, A_{2}(5 / 3)=1.517, A_{3}(5 / 3)=1.301 \text { etc. }
$$

With these definitions the moment $q_{1}^{\rho^{\prime}}$ can be written

$$
\begin{aligned}
q_{1}^{\rho^{\prime}=} \rho_{O} c_{O}{ }^{1+3} & \left\{\left\langle I|| Q_{1}^{(1)}|| I^{\prime}\right\rangle+\frac{1}{2}(1+2)\left\langle I|| Q_{1}^{(2)}|| I^{\prime}\right\rangle\right. \\
& \cdot\left\{H_{1}(K=1)-\gamma \rho_{O}^{2 / 3} H_{1}(\kappa=5 / 3)\right\}
\end{aligned}
$$

The volume integral of the effective density (eq.2.4a) can calculated [12] by a function $F(\varepsilon) \cdot\left(1-\gamma_{\rho} \rho^{2 / 3}\right)=J_{\rho} \cdot / J_{\rho}$

$$
\begin{aligned}
& F(\varepsilon)=1+B_{1} \varepsilon+B_{2} \varepsilon^{2}+B_{3} \varepsilon^{3}+\ldots \\
& B_{1}=3 A_{1}(5 / 3) \alpha \quad B_{2}=\left[\pi^{2}-6 A_{2}(5 / 3)\right] \alpha \\
& B_{3}=2 \cdot\left[3 A_{3}(5 / 3)-A_{1}(5 / 3) \pi^{2}\right] \alpha
\end{aligned}
$$


Using the expansions $H_{1}(\kappa)$ and $F(\varepsilon)$ we modify eq. 1.1

$$
\frac{q_{1}^{u}}{J_{u}}=\frac{q_{1}^{\rho}}{J_{\rho}} \cdot c_{1}
$$

with the correction function

$$
C_{1}=\left|\begin{array}{ll}
1-\gamma \rho_{0} & \frac{\mathrm{H}_{1}(k=5 / 3)}{\mathrm{H}_{1}(k=1)}
\end{array}\right| /\left(F(\varepsilon) \cdot\left(1-\gamma \rho_{0}^{2 / 3}\right)\right)
$$

$\mathrm{C}_{1}$ reduces to 1 , if $\gamma=0$ (density independent) or $\varepsilon=0$ (homogenous distribution with sharp edge). To first order in deformation $(t=1)$ and second order in $\varepsilon$

$$
\begin{aligned}
C_{1}=1 & +(1-1) \cdot A_{1}(k=5 / 3) \alpha \cdot \varepsilon \\
& +(1-1) \alpha \varepsilon^{2}\left[\left(\frac{\pi^{2}}{6}-A_{2}(k=5 / 3)\right)(1+4)-3 A_{1}^{2}(k=5 / 3) \alpha\right]
\end{aligned}
$$

which is independent from the "deformation"

Using this expression in Fig. $1, C_{1}$ is plotted for $\alpha=1.34$ $\left(\gamma=1.9 \mathrm{fm}^{2} \text { and } \rho_{0}=0.165 \mathrm{fm}^{-3}\right)^{1}$ with $\varepsilon=0.54 /\left(1.12 \mathrm{~A}^{1 / 3}\right) \mathrm{fm}$.

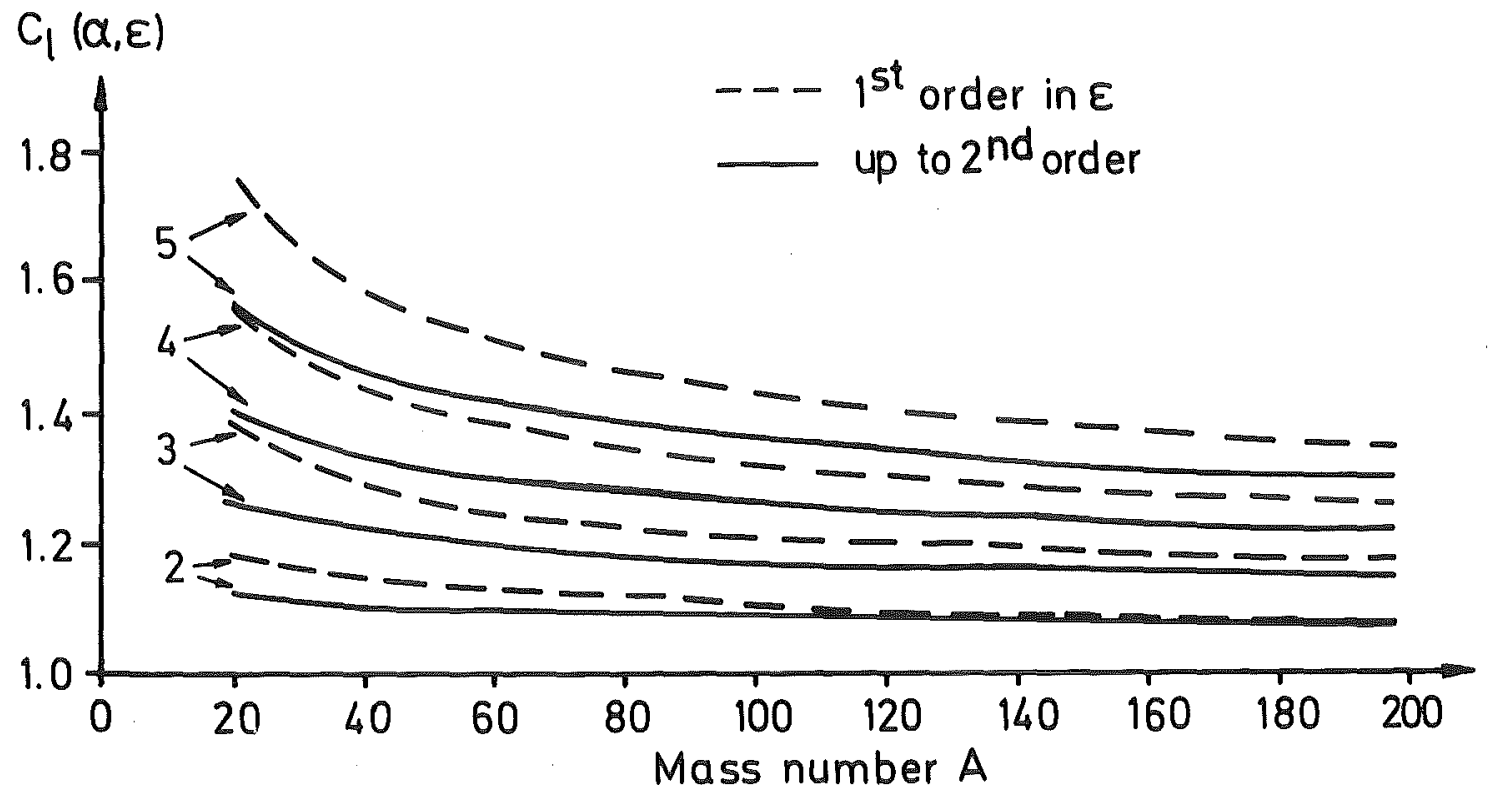

Fig. 1 Correction of the normalized multipole moments of a deformed Fermi distribution due to density dependence 
It is interesting to note that the second order tries to cancel the effect.

\section{Discussion}

Implicit folding model procedures [1-5] have considerably improved the reliability of the results extracted from experimental data and have replaced the crude procedure previously used [16]. However there are only few results with realistic error estimates, and some results suffer on constraints or on the neglect of multiple excitation in the primary (extended) optical model analysis. It has been observed [1], that the extracted moments tend to have slightly larger values when compared to different studies. For light nuclei, in particular for $N=Z$ nuclei, we have to expect that the isoscalar rates do not differ from electromagnetic rates. In fact most of the published results, especially for higher multipolarities, are improved in this sense by the $C_{1}$ correction. Remaining discrepancies may be ascribed to other reasons, e.g. the limits of a first order DWBA when applied for hexadecapole transitions. It should be also emphasized that the results may be influenced by the constraints due to the particular choice of the functional form for the radial shape of the transition potentials, increasingly with higher multipolarities. There are only few studies $[2,4]$ which minimize this influence. Similarly, the correction function $C_{1}$ is expected to become less accurate for larger 1-values as the tails of the transition densities are not very well approximated by derivatives of a Fermi distribution. Due to this fact, that an accurate calculation of the corrections requires a good knowledge of the densities, the implicit folding procedures loose somehow their primary elegance.

Tab. 2 Intrinsic multipole moments of ${ }^{56} \mathrm{Fe}$

\begin{tabular}{|ccccccc|}
\hline$Q_{20}^{\rho^{\prime}}$ & $Q_{20}^{\rho}$ & $Q_{40}^{\rho^{\prime}}$ & $Q_{40}^{\rho}$ & $Q_{20}^{\text {fold }}$ & $Q_{40}^{\text {fold }}$ & $Q_{20}^{\text {elec }}$ \\
{$\left[\mathrm{efm} \mathrm{m}^{2}\right]$} & {$\left[\mathrm{efm} \mathrm{m}^{2}\right]$} & {$\left[\mathrm{efm} \mathrm{m}^{4}\right]$} & {$\left[\mathrm{efm} \mathrm{m}^{4}\right]$} & {$\left[\mathrm{efm} \mathrm{m}^{2}\right]$} & {$\left[\mathrm{efm}{ }^{4}\right]$} & {$\left[\mathrm{efm} \mathrm{m}^{2}\right]$} \\
\hline$+125 \pm 5$ & $114 \pm 4$ & $+615 \pm 100$ & $+469 \pm 75$ & $+100 \pm 4$ & $+500 \pm 90$ & $98 \pm 2$ \\
\hline
\end{tabular}


Table 2 presents a particular example of values of quadrupole and hexadecapole moments as found by $104 \mathrm{MeV} \alpha$-particle scattering and resulting from different types of analysis. The $Q_{10}^{\rho}$ are the values derived by the implicit folding procedure in ref. 1 , $Q_{10}^{\rho}$ are values corrected by the $C_{1}$ factor showing improved agreement with electromagnetic result. The values $Q_{10}^{\text {fold }}$ result from an explicit folding model analysis [1] using a densityindependent Gaussian effective interaction. This interaction, empirically found, describes very well the differential cross sections in the diffraction region, but fails for the backward angles due to the lack of saturation. In fact, it does not reproduce the phenomenologically found volume integral $J_{u}$ of the real interaction. Approximately $J_{u} / J_{v} \simeq A\left(1-\gamma \rho_{0}^{2 / 3}\right) \approx 0.5 \cdot A$ is found. In contrast a density-dependent interaction as given by Friedman et al.[17] e.g. is able to resolve this discrepancy and reproduces the correct value of $J_{u}$ within few percent.

In view of this inconsistency it is somwehat surprising that the rates and moments extracted by the explicit folding procedure with the density-independent interaction rather well agree with electromagnetic results (see also ref.18). This is a consequence of the increased importance of the nuclear surface in inelastic scattering. In fact the density independent effective interaction applied in refs. 1 and 18 has been adjusted by elastic forward scattering on ${ }^{40} \mathrm{Ca}$, thus effectively averaging over the density dependence in the sensitive region. Obviously, such an adequately adjusted interaction is working well in the transition region and provides results consistent with electromagnetic values, even if its saturation properties are wrong. Considering the density-dependent effective interaction of the type as given in ref. 17, the situation seems to be just reversed. Forcing $V_{\text {eff }}$ by the factor $g(\rho)$ (eq. 2.2) to give the correct saturation leads to a too strong interaction at the surface (where $g(\rho)$ is small) as compared to the sucessfull density independent form of $v_{\text {eff }}\left(J_{v}\right.$ density $d \cdot / J_{v}^{O}$ $>1$ for $\left.r>c_{0}\right)$. This possible deficiency of $g(\rho)$ affects mainly the inelastic transition potentials, and indeed somewhat strange results (reduced rates) are found when applying density-dependent 
effective interactions of the type (eq. 2.1) in inelastic folding model analyses. [19] Most recently [9] even in elastic scattering some inadequacy of the form factor $g(\rho)$ (eq.2.2) has been observed.

This uncertainty in the correct form of $g(\rho)$ may also influence the correction factor $C_{1}$. This remark includes some warning about the significance of differences of the neutron and proton components of the deformation as quoted in ref. $5 \mathrm{e.g}$. We conclude that comparative studies of electromagnetic and hadronic transition rates are of interest not only with respect of their nuclear structure information, but also by revealing details of the density dependence of the nucleus-nucleus interaction.

The authors would like to thank Dr. N.K. Ganguly and Dr. J. Albihski for encouraging discussions. 


\section{References}

1 H. Rebel, Z. Phys. A277 (1976) 35

2 H. Rebel, R. Pesl, H.J. Gils and E. Friedman Nuc 1. Phys. $\AA 368$ (1981) 61

3 G.J. Wagner, P. Grabmayr and H.R. Schmidt Phys. Lett. 113B (1982) 447

4 V. Corcalciuc, H. Rebel, R. Pesl and H.J. Gils

J. Phys. G: Nucl. Phys. 9 (1983) 177

5 R.S. Mackintosh, Nucl. Phys. $\underline{\text { 266 }}$ (1976) 379

6 G.R. Satchler, J. Math. Phys. 13 (1972) 1118

7 F. Friedman, H.J. Gils, H. Rebel and Z. Majka Phys. Rev. Lett. 41 (1978), 1220

8 Z. Majka, H.J. Gils and H. Rebel

Z. Phys. A288 (1978) 139 - Acta Phys. Polonica B11 (1980) 227

9 H.J. Gils, Phys. Lett. B (submitted) - KfK 3555 (1983)

10 D.K. Srivastava, preprint May 1983

11 D.K. Srivastava, Phys. Lett. 122B (1983) 18

12 D.K. Srivastava, Phys. Lett. 113B (1982) 353

13 E. Friedman, H.J. Gils, H. Rebel and R. Pesl, Nucl. Phys. A363 (1981) 137

14 H.J. Gils, H. Rebel, G. Nowicki, A. Ciocânel, D. Hartmann, $H$. Klewe-Nebenius and $K$. Wisshak Journ. Phys. G: Nucl. Phys. 3 (1975) 344

15 D.K. Srivastava, Phys. Lett. 112B (1982) 289

16 A.M. Bernstein, Adv. Nucl. Phys. 3 (1969) 325, eds. M. Barenger and $\mathrm{E}$. Vogt, Plenum Press 1969

17 E. Friedman, H.J. Gils, H. Rebel and Z. Majka Phys. Rev. Lett. 41 (1978) 1220

18 H.J. Gils and H. Rebel, Z. Phys. A274 (1975) 259

19 R. Pesl, H.J. Gils, H. Rebel, E. Friedman, J. Buschmann, H. Klewe-Nebenius, S. Zagromski, KfK-3378 (1983) Z. Phys. 1313 (1983) (in press) 
Appendix

We present numerical results based on a density distribution

$$
\begin{aligned}
& \rho_{0}^{\kappa} \cdot f(\kappa) \text { with } \\
& f(\kappa)= 1 /\left[1+\exp ((r-c) / a]^{k}\right. \\
& c_{0}=1.115 \cdot A^{1 / 3}-0.53 \cdot A^{-1 / 3} \mathrm{fm} \\
& a \quad=0.57 \\
& \kappa \quad=1 \text { and } \kappa=5 / 3 .
\end{aligned}
$$

Choosing

$$
\begin{aligned}
& \left\langle 0|| Q_{1}{ }^{(1)}|| I\right\rangle=\delta_{\lambda I}(-)^{I} B_{1} \\
& \left\langle 0|| Q_{1}{ }^{(2)}|| I\right\rangle=B_{1}^{2}\langle 1100 \mid I 0\rangle / \sqrt{2} \\
& \text { with } B_{1}=1
\end{aligned}
$$

same quantities of interest and the correction factor $\mathrm{C}_{1}=$ $\left(q_{p}, / J_{\rho},\right) /\left(q_{\rho} / A\right)$ are tabulated for $1=2,3,4,5$, in addition to the radial moments $y(k)$ and $z(k)$. We note that the tabulated values result from numerical integration of the revelant expressions and allow to check the numerical precision of the algebraic approximations introduced in sect. 3. Tab. A 1 shows the error range for $A \in[20,206]$ of the approximations represented by

$$
\Delta \dot{y}_{1}=\frac{y_{1} \text { (algebr.) }=y_{1} \text { (exact.) }}{y_{1} \text { (exact) }} \cdot 100
$$

e.g.. $\Delta C_{1}$ refers to the error when $C_{1}$ is calculated by (3.12) up to high orders in $\varepsilon$.

\begin{tabular}{|c|c|c|c|c|c|}
\hline 1 & $\Delta y_{1}(1)$ & $\Delta y_{1}(5 / 3)$ & $\Delta z_{I}(1)$ & $\Delta z_{1}(5 / 3)$ & $\Delta C_{1}$ \\
\hline 2 & {$[0.01,1.66]$} & {$[0.22,0.42]$} & {$[0.07,2.68]$} & {$[0.25,0.47]$} & {$[0.53,2.35]$} \\
3 & {$[0.01,3.21]$} & {$[0.27,0.51]$} & {$[0.09,4.65]$} & {$[0.33,0.47]$} & {$[0.51,2.07]$} \\
4 & {$[0.01,6.00]$} & {$[0.32,0.48]$} & {$[0.11,7.77]$} & {$[0.40,0.41]$} & {$[0.51,1.84]$} \\
5 & {$[0.015,10.58]$} & {$[0.37,0.48]$} & {$[0.12,12.81]$} & {$[0.47,0.58]$} & {$[0.52,1.64]$} \\
\hline
\end{tabular}

Tab. A 1 Range of errors in percent for $A \in[20,206]$ when using the power series expansions of sect. 3 . 
Tab. A2

Central density and normalized multipole

moments $\left(B_{1}=1\right)$

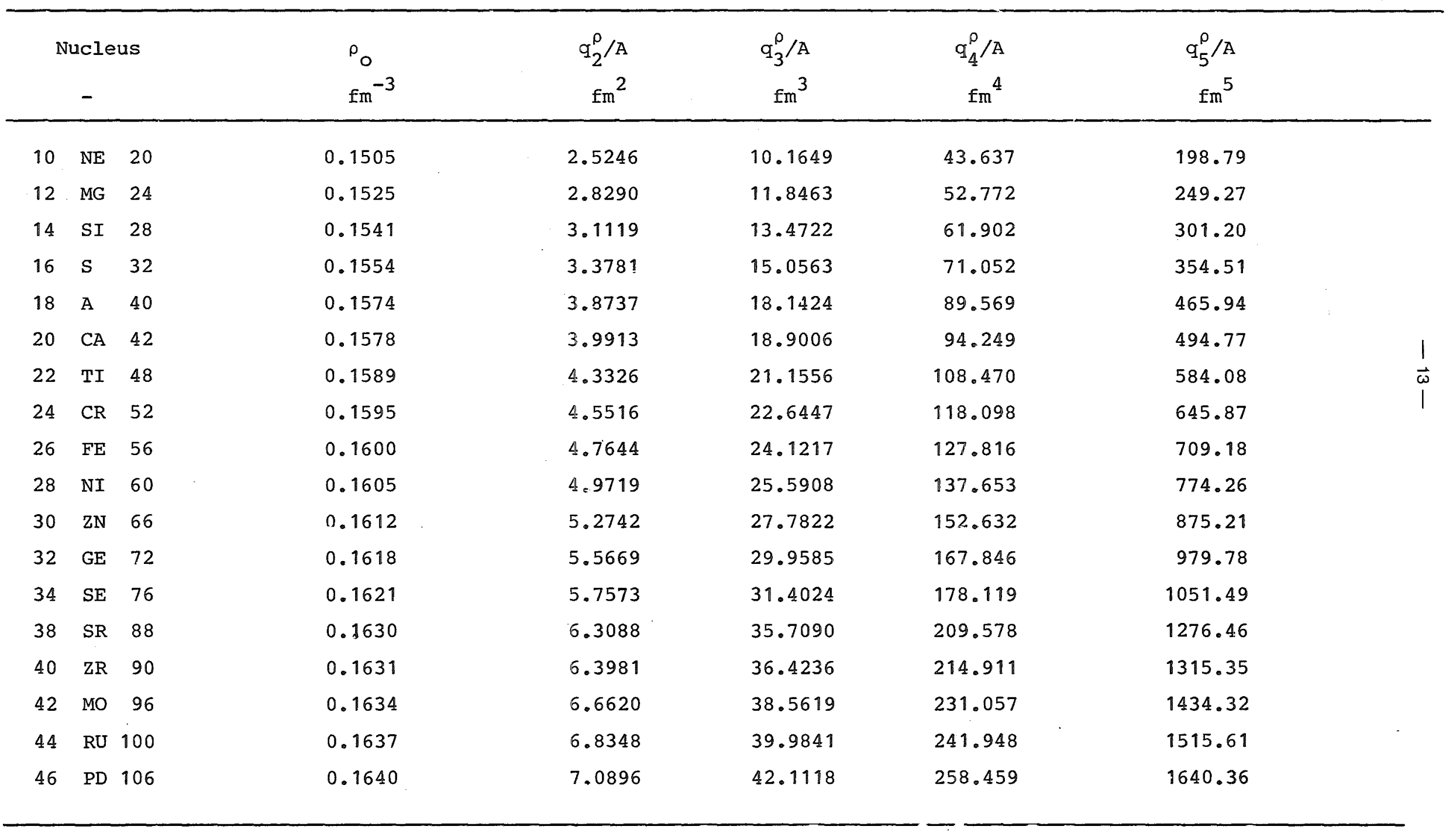


Tab. A: 2 (contd.)

\begin{tabular}{|c|c|c|c|c|c|}
\hline $\begin{array}{c}\text { Nucleus } \\
-\end{array}$ & $\begin{array}{l}\rho_{0} \\
\mathrm{fm}^{-3}\end{array}$ & $\begin{array}{l}q_{2}^{\rho} / A \\
\mathrm{fm}^{2}\end{array}$ & $\begin{array}{l}q_{3}^{\rho} / A \\
\mathrm{fm}^{3}\end{array}$ & $\begin{array}{c}q_{4}^{\rho} / A \\
\mathrm{fm}^{4}\end{array}$ & $\begin{array}{l}q_{5}^{\rho} / A \\
\mathrm{fm}^{5}\end{array}$ \\
\hline $48 \mathrm{CD} 110$ & 0.1642 & 7.2568 & 43.5275 & 269.586 & 1725.44 \\
\hline 50 SN 116 & 0.1644 & 7.5037 & 45.6461 & 286.443 & 1855.76 \\
\hline $\begin{array}{lll}52 & \text { TE } & 124\end{array}$ & 0.1648 & $7=8267$ & 48.4641 & 309.238 & 2034.71 \\
\hline 56 BA 136 & 0.1652 & 8.2972 & 52.6722 & 344.074 & 2313.83 \\
\hline 60 ND 144 & 0.1654 & 8.6033 & 55.4778 & 367.713 & 2506.90 \\
\hline 62 SM 148 & 0.1655 & 8.7542 & 56.8759 & 379.655 & 2605.52 \\
\hline $\begin{array}{lll}78 & \text { PT } & 194\end{array}$ & 0.1666 & 10.4014 & 72.8693 & 522.413 & 3834.80 \\
\hline 80 HG 200 & 0.1667 & 10.6059 & 74.9458 & 541.728 & 4007.63 \\
\hline 82 PB 206 & 0.1668 & 10.8084 & 77.0206 & 561.194 & 4183.25 \\
\hline
\end{tabular}




\begin{tabular}{|c|c|c|c|c|c|c|c|c|}
\hline A & $y_{2}(1)$ & $z_{2}(1)$ & $y_{3}(1)$ & $z_{3}(1)$ & $y_{4}(1)$ & $z_{4}(1)$ & $y_{5}(1)$ & $z_{5}(1)$ \\
\hline 20 & $.504918 E+02$ & $.740246 \mathrm{k}+02$ & (cos a & (1) & $872 r s 0 t+42$ & $.103 \angle 7 / E+04$ & $.397546 E+44$ & $912705 E+04$ \\
\hline 26 & $.678962 E+02$. & $.905663 E+03$ & $.284590 E+03$ & $.504691 E+03$ & $.126050 E+04$ & $.250993 E+04$ & $.598248 \mathrm{E}+\cup 4$ & $.128548 E+05$ \\
\hline 28 & $.879325 E+02$ & $.130305 E+03$ & $.371229 E+03$ & $.090090 E+0 S^{-}$ & $.173524 E+04$ & $.355799 E+04$ & $.843346 E+04$ & $.788005 E+05$ \\
\hline 32 & $.108099 \mathrm{E}+03$ & $.11<507=* 03$ & $.487801 E+0 S$ & $.902740 E+03$ & $.227508 \mathrm{E}+04$ & $.480080 E \div 04$ & $.993443 E * U 5$ & $.062905 E * 05$ \\
\hline 40 & $.154940 E+05$ & $.254500 E+03$ & $.723096 E+03$ & $.140953 E+04$ & $.358270 E+04$ & $.789530 E \$ 04$ & $.986376 E * 45$ & $.4526005+05$ \\
\hline 62 & $: 16763$ SE+0S & $.27 r 020 E+03$ & $.79 د 825 E+03$ & $.73530 \% E+04$ & $.395844 E+04$ & $879879 E \propto 04$ & $.207804 E \$ 05$ & $0509494 E+05$ \\
\hline 48 & $.207967 E+0 S$ & $.340905 t+03$ & $.901547 E+04$ & $.202454 E+04$ & $.520052 E+04$ & $.118575 E \div 05$ & $.2803577_{E+45}$ & $.7057 j 8 E+05$ \\
\hline 52 & $236684 \mathrm{E}+0 \mathrm{~S}$ & $.400478_{t+03}$ & $.11 r 75 Z_{E}+04$ & $.257279 \mathrm{E}+04$ & $.69410^{8} \mathrm{E}+U^{4}$ & $.941425 E+05$ & $.3358>4 E+45$ & $.858187 E+05$ \\
\hline 56 & $.266807 E+03$ & $.454832 E+03$ & $.130082 E+04$ & $.274702 E+04$ & .79357 IIE+v4 & $.966705 E=05$ & $.3971395+45$ & $.1028 \div 9 t=06$ \\
\hline 60 & $.298312 E+0 S$ & $.511945 E=03$ & $.152345 E+04$ & $.314951 E+04$ & $.825427 E+0^{4}$ & $.194310 E=05$ & $.066554 E+05$ & $929 / 22 E+06$ \\
\hline 66 & $.348099 E+0 S$ & $.00<0060 \rightarrow 03$ & $.985302 E+04$ & $.380370 E+04$ & $.90075 Y E+02$ &, $240208 E+05$ & $.577636 E-U 5$ & $.1537000 \% 06$ \\
\hline 72 & $: 600879 E+05$ & $.694222 z+03$ & $.213701_{E}+04$ & $.459690 E+04$ & $.12084 Y E+U 2$ &.$\angle 91525 E \otimes 05$ & $.705641 E \propto 05$ & $.1903045+00$ \\
\hline 7.6 & $.437556 E+0 S$ & $.76075 D E+03$ & $.238059 g_{E}+04$ & $.502621 E+04$ & .135SIUE + DV & $.328816 E+05$ & $.799935 E+45$ & $0 \leqslant 17419 E+06$ \\
\hline 88 & $.555170 E+03$ & $.984129 t+03$ & $.374240 E+04$ & $.071543 E+04$ & $.984428 E+U\rangle$ & $.455999 E+05$ & $.112328 E+U 6$ & $\$ 12074 E+06$ \\
\hline 90 & $.575826 \mathrm{E}+0 \mathrm{~S}$ & $.90<246 E+04$ & $.32 r 812 E+04$ & $.702040 E+04$ & $.1934 \angle U E+U>$ & $.479475 E \div 05$ & $.19 .8382 E \otimes U 6$ & $.529904 E+06$ \\
\hline 96 & $.639549 E+03$ & $.114095 E+v 4$ & $.374796 E+04$ & $.747559 E+04$ & .22181DE+U2 & $.353870 E \oplus 05$ & $.137645 E * U 6$ & $. \triangle 870 S 6 E+00$ \\
\hline 0 & $.683479 \mathrm{E}+03$ & $.12<284 E+04$ & $.394849 E+04$ & $.804600 E+04$ & $.249448 E+02$ & $.000850 E>05$ & $.151569 E * U 6$ & $.428278 E+06$ \\
\hline 106 & $.751501 E+0 S$ & $.154991 E+04$ & $.440386 E+04$ & $.9 \% 0170 E+04$ & $.273460 E+U 3$ & $.091429 E+05$ & $.173 B 78 E \oplus \cup G$ & $.496901 E+06$ \\
\hline 190 & $.798249 \mathrm{E}+0 S$ & $.145743 E+U 4$ & $.478802 E+04$ & $.904391 E+05$ & $.290343 E+03$ & $.151294 E \div 05$ & $.1897998 E V 6$ & $.342750 E+06$ \\
\hline 196 & $.870430 E+03$ & $.151280=004$ & $.524495 E+04$ & $.115951 E+05$ & $.332274 E+02$ & $.846333 E+05$ & $.295208 E+46$ & $.0195055+06$ \\
\hline 124. & $.97045 .7 E+03$ & $.1700806=04$ & $.600955 E+04$ & $.132304 E+05$ & $.383452 E+05$ & $.483079 E \div 05$ & $.252304 E \otimes U 6$ & . IS17SOE\& 06 \\
\hline 136 & $: 112841 E+04$ & $.203876 E+04$ & $.710410 E+04$ & $.738838 E+05$ & .46794 UE $+0 D$ & $.127007 E+06$ & $.394681 E * 06$ & $.922054 E+06$ \\
\hline 944 & $.123887 E+04$ & $.220763 E+04$ & $.798880 E+04$ & $.977804 E+45$ & $.52930(t+0)$ & $.137629 E+06$ & $.360946 E \$ 06$ & $.1068185+07$ \\
\hline 148 & $.129561 E+04$ & $.2515125+04$ & $.847103 E+04$ & $.187778 E+U b$ & $.56788 Y E+U 2$ & $.146393 E+06$ & $.385617 \mathrm{E}+U \mathrm{G}$ & $.794009 E+07$ \\
\hline 194 & $.201780 \mathrm{E}+04^{\circ}$ & $.3 r>032 E+04$ & us & $.3 \angle 099 U E+U S^{\circ}$ & $=+40$ & 2698 & - $7439>1 E+\cup 6$ & $.225700 E+07$ \\
\hline 100 & $.212119 E+04$ & $905+04$ & .94 & $.340908 E+U S$ & .1083 & $0889728 E+06$ & $.801525 E+U 6$ & $843817 E+07$ \\
\hline 00 & $.222654 E+04$ & $.47495>x+04$ & $.150002 E+05$ & $.301540 E+U S$ & .1950 OEE +00 & $.304162 E \otimes 06$ & $.861749 E+46$ & $0662805 E+07$ \\
\hline
\end{tabular}

Tab A 3 Radial moments $y_{1}(1)$ and $z_{1}(1)$ 


\begin{tabular}{|c|c|c|c|c|c|c|c|c|}
\hline A & $y_{2}(5 / 3)$ & $z_{2}(5 / 3)$ & $y_{3}(5 / 3)$ & $z_{3}(5 / 3)$ & $y_{4}(5 / 3)$ & $z_{4}(5 / 3)$ & $y_{5}(5 / 3)$ & $z_{5}(5 / 3)$ \\
\hline 20 & $.736489 \mathrm{E}+02$ & $463874 t+02$ & $.840 r r B E+U 2$ & .7014JלE+US & . 295 rudetus & $.020720 E 003$ & $.108186 E \$ \forall 46$ & $1<92003 E \leqslant 04$ \\
\hline 34 & $.357633 E+02$ & $.050_{498}=+02$ & $.122470_{E}+U S$ & $.2 ' 104$ I E $+U 3$ & $.45002 y E+02$ & $.912820 E \oplus 04$ & $.1713 r 7 E \& U 4$ & $.077856 E=04$ \\
\hline 28 & $.473218 E+02$ & $.88 \angle 9025=02$ & $.970803 E+03$ & $379848 E+43$ & $.044<4>E+0 S$ & $.164503 E \$ 04$ & $.253500 E \& 46$ & $.723784 E+06$ \\
\hline 32 & $.602697 E+02$ & $.115021=+03$ & $.222654 \mathrm{E}+03$ & $50817 b_{E+U 3}$ & $.87848 \% E+U د$ &,$\angle 28900 E=04$ & $.356525 E \$ 44$ & $.7037395+05$ \\
\hline 40 & $.901513 E+02$ & $.17<5560+03$ & $.358346_{E}+03$ & $.824405 E+03$ & $.148 Y^{\prime} Y_{E}+U^{4}$ & $.395474 E \odot 04$ & $.032746 E \otimes 04$ & $.989622 E+05$ \\
\hline 42 & $.984240 E+02$ & $.988950 E+03$ & $.390825 E+03$ & $.910925 E+U S$ & $.1658 /(\mathrm{f}=04$ & $.443227 \%=04$ & .797876 EQ4Q &.$<767465+05$ \\
\hline 48 & $.125101 E+05$ & $.24 \angle 0095 * 03$ & $.522790 \mathrm{E}+03$ & $.122909 E+04$ & $.226890 \mathrm{E}+\mathrm{U}^{4}$ & $.015892 E \times 06$ & $.91406 E \approx+05$ & .5901795005 \\
\hline 52 & $: 144398 E+0 S$ & $.280590 E+03$ & $.610607 E+03$. & $.745908 E+04$ & .27SYSSE*U4 & $.745970 E \propto 06$ & $.924049 E * 45$ & $.585948 E+05$ \\
\hline 56 & $.964876 E+0 S$ & $.327471 t+03$ & $.727020 E+03$ & $.170120 E+04$ & $.32582 \pi\left(E+U^{4}\right.$ & $.049488>E \div 040$ & $.951581 E \& 45$ & .0706035005 \\
\hline 60 & $.186590 E+03$ & $.304825 E+03$ & $.83<870 E+03$ & $.947247 E+04$ & $.38320<E+0^{4}$ & $.103682 E+05$ & $.981525 E \$ 45$ & $.267306 E \otimes 05$ \\
\hline 66 & $.22108^{4} E+03$ & $.454248 E+03$ & $.107527 E+04$ & $.249797 E+04$ & $i^{479}(4) E+0^{4}$ & $.133247 E+05$ & $.232994 E+45$ & $.736508 E \otimes 05$ \\
\hline 72 & $.258940 E+05$ & $.508826=+03$ & $.921042 E+04$ & $=241932 E+04$ & $.589092 E \otimes 04$ & $.104023 E+05$ & $.292795 E 045$ & $.4500795 \$ 05$ \\
\hline 76 & $.284208 E+03$ & $.5673476+03$ & $.950099 E+04$ & $.3266 \% 9 E+04$ & $.6692 Z 20 E+04$ & $.187725 E+05$. & $.337546 E \$ 05$ & $.1070 y 2 E \$ 06$ \\
\hline 88 & $.368715 E+0 S$ & $.737984 E+03$ & $.184328 E+04$ & $.446908 E+04$ & $.940354 E+U 4$ & $.267948 E \propto 05$ & $.696857 E \$ 05$ & $.760 \$ 18 E \$ 06$ \\
\hline 90. & $.38370 S E+0 S$ & $.76<290 k+03$ & $.199360 E+04$ & $=407900 E+04$ & .99800 CE $\$ 04$ & $.2282958 E+05$ & $.527235 E \nrightarrow 05$ & $04 E+06$ \\
\hline 96 & $.430182 E+0 S$ & $.850342 E \div 03$ & $.221054 E+04$ & $.536608 E+04$ & $.11020 Y E+U 2$ & $.330897 E=05$ & $.025347 E+05$ & $.003048 E \$ 06$ \\
\hline 100 & $.462416 E+03$ & $.921021 k+03$ & $.24 U 597 E+04$ & $.585084 E+04$ & $.128008 E+02$ & $.365300 E \otimes 05$ & $.096790 E \$ 05$ & $.226858 E+06$ \\
\hline 06 & $: 512610 E+03$ & $.10<334 E=04$ & $.277317 E+04$ & $.601882 E+04$ & $.14702 د E+U D$ & $.420700 E+05$ & $.813173 E 40 \overline{05}$ & .2657791400 \\
\hline 10 & $.547286 E+0 S$ & $.1043600=06$ & $.292204 E+04$ & $.715813 E+U_{4}$ & $.96039<E+U 2$ & $0460189 E \$ 05$ & $.897956 \mathrm{E} * 05$ & $.293915 E+06$ \\
\hline 116 & $.609094 E+03$ & $.920283 E+04$ & $.321349 E+0.4$ & $.800821 E+U_{4}$ & $.18204 Y E+0 D$ & $.523342 E * 05$ & $.103506 E \$ U 6$ & $.5393>55+00$ \\
\hline 124 & $. A 76138 E+03$ & $.753523 E+04$ & $.373901 E+04$ & $=9 \angle 1800 E+04$ & $.213<4 r E+U 2$ & $.013098 E=05$ & $.123348 E \otimes U 6$ & .0070475006 \\
\hline 136 & $: 795632 E+03$ & $.954808 E+04$ & $.453236 E+04$ & $.112001 E+U S$ & $.263212 E+U 2$ & $.109003 E+0 S$ & $.157746 E 406$ & $.3233 ? 9 E+06$ \\
\hline 144 & $.879818 \dot{E}+0 S$ & $.970930 E+06$ & $.57<484 E+04$ & $=926327 E+05$ & $.304704 E+U S$ & $.882465 E+05$ & $.983711 E \varangle U 6$ & $.019278 E+06$ \\
\hline 148 & $.923246 E+03$ & $.985706 E+04$ & $.54 \angle 420 E+04$ &. TSBBTSE+US & $.324350 E+U 2$ & $.443490 E \div 05$ & $.997643 E+06$ & $.038573 E=06$ \\
\hline 194 & $-148430 E+04$ & $00069 t+04$ & $4 Y 979 E+04$ & $56120 E+05$ & $Y E+U D$ & $81341 E \ngtr 06$ & $46 E+U 6$ & $.137532 E+07$ \\
\hline 200 & $.156500 E+04$ & $.370045 t+04$ & $.901177 E+05$ & $.231672 E+0 S$ & $.663451 E+U 2$ & $=195164 E \otimes 06$ & $.642203 E+U 6$ & . 1.69418E\&07 \\
\hline 200 & $.9648776+04$ & $.352592 E+04$ & $.901357 E+05$ & $.20773 U E+U S$ & $P 1231 Y E \in U 2$ & $\angle U Y S B S E \otimes 0 S$ & . $478003 E+U 6$ & $.9014945+07$ \\
\hline
\end{tabular}

Tab A 4 Radial moments $y_{1}(5 / 3)$ and $z_{1}(5 / 3)$ 
Tab. A 5

Correction quantities

\begin{tabular}{|c|c|c|c|c|c|c|c|c|}
\hline & NUCLEUS & $\mathrm{K} / \mathrm{A}$ & $\mathrm{J}_{\rho^{*}} / \mathrm{A}$ & $c_{2}$ & $c_{3}$ & $\mathrm{C}_{4}$ & $c_{5}$ & \\
\hline & - & $\mathrm{fm}^{-2}$ & - & - & - & - & - & \\
\hline & $\mathrm{NE} 20$ & 0.172 & 0.674 & 1.078 & 1.151 & 1.213 & 1.266 & \\
\hline 12 & MG 24 & 0.178 & 0.661 & 1.081 & 1.155 & 1.221 & 1.278 & \\
\hline & SI 28 & 0.184 & 0.650 & 1.082 & 1.158 & 1.226 & 1.285 & \\
\hline 16 & s 32 & 0.189 & 0.641 & 1.082 & 1.159 & 1.229 & 1.291 & \\
\hline 18 & A 40 & 0.197 & 0.626 & 1.083 & 1.160 & 1.232 & 1.297 & $\vec{v}$ \\
\hline 20 & $C A \quad 42$ & 0.198 & 0.623 & 1.083 & 1.160 & 1.232 & 1.298 & I \\
\hline 22 & TI 48 & 0.203 & 0.614 & 1.082 & 1.160 & 1.233 & 1.299 & \\
\hline 24 & $\mathrm{CR} 52$ & 0.206 & 0.609 & 1.082 & 1.160 & 1.233 & 1.300 & \\
\hline 26 & FE 56 & 0.208 & 0.605 & 1.081 & 1. 159 & 1.232 & 1.300 & \\
\hline 28 & NI 60 & 0.210 & 0.601 & 1.081 & 1.158 & 1.232 & 1.300 & \\
\hline 30 & $\mathrm{ZN} 66$ & 0.213 & 0.595 & 1.080 & 1.157 & 1.231 & 1.300 & \\
\hline 32 & GE 72 & 0.216 & 0.590 & 1.080 & 1.156 & 1.229 & 1.299 & \\
\hline 34 & SE 76 & 0.218 & 0.587 & 1.079 & 1.156 & 1.229 & 1.298 & \\
\hline 38 & SR 88 & 0.222 & 0.579 & 1.078 & 1.153 & 1.226 & 1.295 & \\
\hline 40 & ZR 90 & 0.222 & 0.577 & 1.078 & 1.153 & 1.225 & 1.295 & \\
\hline 42 & MO 96 & 0.224 & 0.574 & 1.077 & 1.152 & 1.224 & 1.293 & \\
\hline 44 & RU 100 & 0.225 & 0.572 & 1.077 & 1.151 & 1.223 & 1.292 & \\
\hline & PD 106 & 0.227 & 0.569 & 1.076 & 1.150 & 1.222 & 1.291 & \\
\hline
\end{tabular}


Table A 5 (contd.)

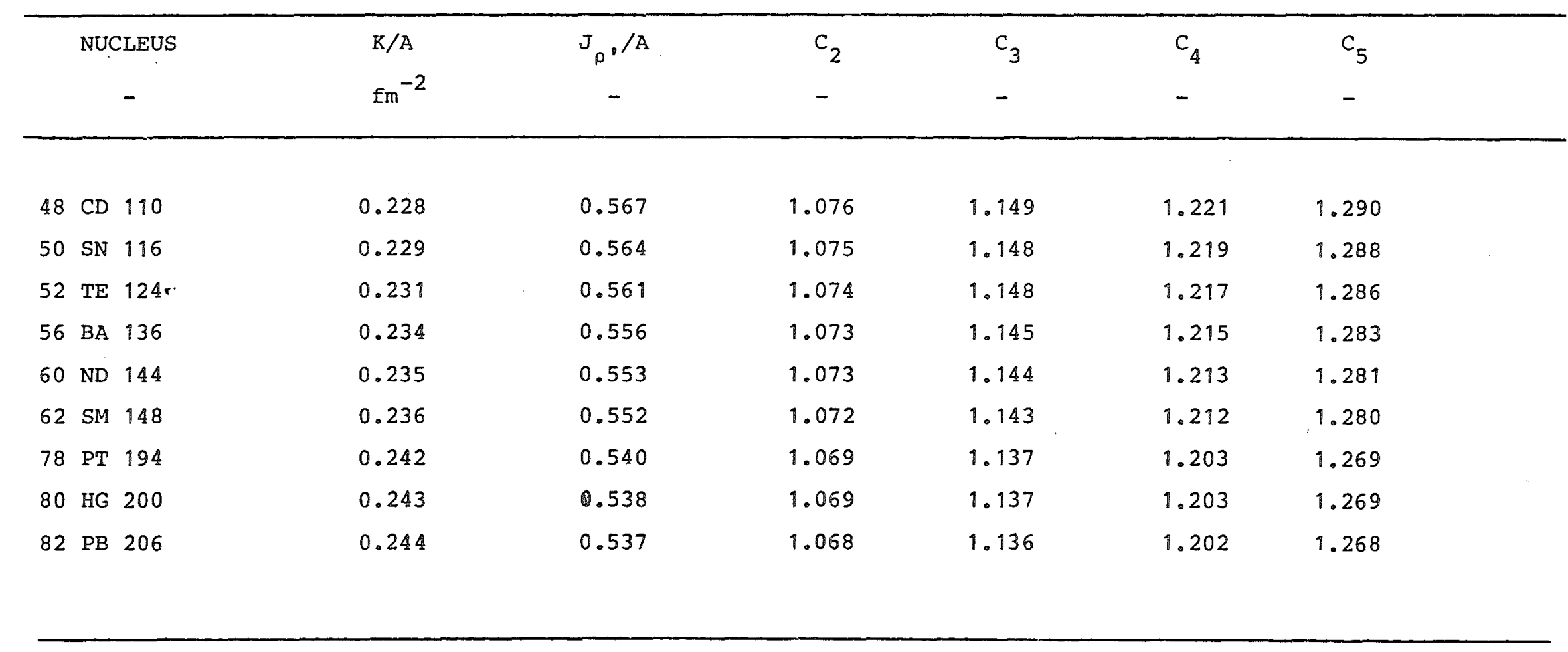

